\title{
Charge generation in organic solar cell materials studied by terahertz spectroscopy
}

\author{
M. Scarongella, ${ }^{\mathrm{a} b \mathrm{~b}}$ J. C. Brauer, ${ }^{\mathrm{a}}$ J. D. Douglas, ${ }^{\mathrm{c}}$ J. M. J. Fréchet, ${ }^{\mathrm{c}, \mathrm{d}}$ and N. Banerji ${ }^{\mathrm{a} *}$ \\ ${ }^{\mathrm{a}}$ Department of Chemistry, University of Fribourg, Chemin du Musée 9, CH-1700, Switzerland; ${ }^{\mathrm{b}}$ Institute of \\ Chemical Sciences \& Engineering, Ecole Polytechnique Fédérale de Lausanne (EPFL), SB ISIC GR-MO, \\ Station 6, CH-1015 Lausanne, Switzerland; ${ }^{\mathrm{c}}$ Department of Chemistry, University of California, 727 Latimer \\ Hall, Berkeley, California 94720-1460, USA; ${ }^{\mathrm{d} K i n g}$ Abdullah University of Science and Technology \\ (KAUST), Thuwal, 23955-6900, Saudi Arabia
}

\begin{abstract}
We have investigated the photophysics in neat films of conjugated polymer PBDTTPD and its blend with PCBM using terahertz time-domain spectroscopy. This material has very high efficiency when used in organic solar cells. We were able to identify a $\mathrm{THz}$ signature for bound excitons in neat PBDTTPD films, pointing to important delocalization in those excitons. Then, we investigated the nature and local mobility (orders of magnitude higher than bulk mobility) of charges in the PBDTTPPD:PCBM blend as a function of excitation wavelength, fluence and pump-probe time delay. At low pump fluence (no bimolecular recombination phenomena), we were able to observe prompt and delayed charge generation components, the latter originating from excitons created in neat polymer domains which, thanks to delocalization, could reach the PCBM interface and dissociate to charges on a time scale of $1 \mathrm{ps}$. The nature of the photogenerated charges did not change between $0.5 \mathrm{ps}$ and $800 \mathrm{ps}$ after photo-excitation, which indicated that the excitons split directly into relatively free charges on an ultrafast time scale.
\end{abstract}

Keywords: Organic solar cells, conjugated polymers, charge generation, mobility, terahertz spectroscopy

\section{INTRODUCTION}

The efficiency of organic solar cells containing the PBDTTPD:PCBM blend as active material is up to $8.5 \%$ ) [1]. A lot of effort has recently been invested on PBDTTPD, showing the importance in understanding the process of charge generation. Control of morphology, charge-trapping, PCBM percolation, and the effect of excess excitation energy, have been amongst the most studied issues, using photoluminescence and absorption techniques [2-5]. Here, we specifically address the understanding of the characteristic photophysics of neat PBDTTPD films and behind the charge separation in PBDTTPD:PCBM blends, as studied by terahertz (THz) spectroscopy.

$\mathrm{THz}$ time domain spectroscopy is very sensitive to detect the contribution of free charges with femtosecond time resolution. Another strength of the THz technique is to have access not only to the amplitude but also to the phase shift of the THz field as it passes through the photo-excited sample, so that is possible to extract the complex conductivity and from this to obtain the real and the imaginary parts. This allows to distinguish between the presence of bound charges (in excitons or charge transfer states) and free charges [6,7]. Moreover, $\mathrm{THz}$ time domain spectroscopy allows also to detect the intrinsic mobility of generated charges, which is conceptually related to the local mobility of charges: this value of mobility is different from the one obtained applying an external electric field, which gives a long range mobility between the two electrodes [8].

Nevertheless, THz time domain spectroscopy has been a less used technique to study the process of charge generation in conjugated polymer blends, because measurements are technically difficult and yield very weak signals. It has been used to find the mobility of charges (electrons and holes) in P3HT, P3HT:PCBM and PTB7:PCBM [9],[10],[11, 12].

*natalie.banerji@unifr.ch; http://www.chem.unifr.ch/banerji

Organic Photovoltaics XVI, edited by Zakya H. Kafafi, Paul A. Lane, Ifor D. W. Samuel, Proc. of SPIE Vol. 9567, 95670M · (C) 2015 SPIE · CCC code: 0277-786X/15/\$18 · doi: 10.1117/12.2187473 
Moreover, the charge transport dynamics when changing the molecular weight in APFO-3:PCBM blends [13], the interchain interactions in polymer films and solutions (related to the polarizability) [14], and the dynamics of photo generation in MEH-PPV were also investigated [15]. However, most of these measurements have been done using very high excitation fluencies, where phenomena of bimolecular recombination are enhanced and mask the real dynamics of the process of charge generation. We have examined neat PBDTTPD films and blends with PCBM, using THz time domain spectroscopy, varying the fluence around $30 \mu \mathrm{J} / \mathrm{cm}^{2}$, in order to suppress the bimolecular recombination and annihilation phenomena as much as possible. We examined also the photoconductivity spectra at $0.5 \mathrm{ps}$ and $800 \mathrm{ps}$ in the range between $0.2-2 \mathrm{THz}(0.8-8 \mathrm{meV})$. The probe in this wavelength range is at too low energy to be able to promote any electronic transitions and it is below any excitation binding energy or exciton resonances. However, it is sensitive to the dielectric polarization of excitons and to the motion of polarons.

\section{METHODOLOGY}

Samples. PBDTTPD (poly(benzo[1,2-b:4,5-b0]dithiophene-alt-thieno[3,4-c]pyrrole-4,6-dione) was synthesized according to previously published procedures [16]. Its chemical structure is shown in Figure 1. The PBDTTPD polymer bears alkyl side-chains on both the TBD (thienopyrroledione) and BDT (benzodithieophene) units, which are necessary for solubility. Side-chains of different lengths can be attached on the TPD unit, strongly affecting solar cell efficiency. In this chapter, we use the polymer with the side-chain designated as P3 $\left(\mathrm{M}_{\mathrm{n}}=27 \mathrm{kDa}\right.$, PDI $\left.=1.9\right)$. The PBDTTPD and PBDTTPD:PCBM mixture (1:1.5 weight ratio) was dissolved in anhydrous chlorobenzene at a $10 \mathrm{mg} / \mathrm{mL}$ polymer concentration inside a glovebox. It was stirred for 48 hours at $100-120^{\circ} \mathrm{C}$. The hot solutions were drop-cast on quartz substrates (film thickness $>500 \mathrm{~nm}$ ).

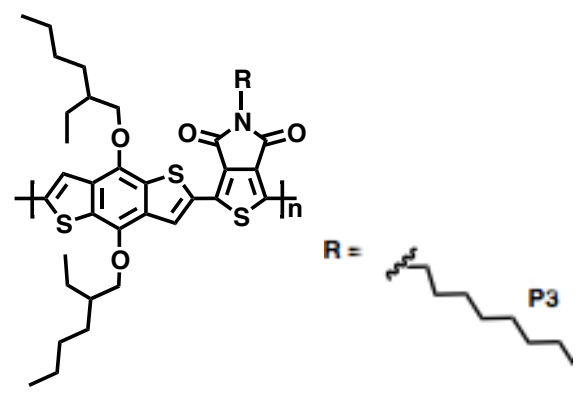

Figure 1 Chemical structure of PBDTTPD.

THz spectroscopy. In the THz setup, the main laser source was an amplified Ti:Sapphire femtosecond laser (LIBRA, Coherent), which provided pulses with a central wavelength of $800 \mathrm{~nm}$, pulse energy of $4.5 \mathrm{~mJ}$ and a temporal width of about 45 fs. We use an Optical Parametric Amplifier (Opera Solo, Coherent) in order to generate the visible excitation wavelengths. To obtain $530 \mathrm{~nm}$, we used a configuration called the Sum Frequency of the Signal (SFS), where the signal was generated by parametric amplification, overlapping a part of the $800 \mathrm{~nm}$ laser fundamental with a temporally chirped white light beam on a BBO crystal. The photon flux of the pump was changed from a minimum of $9 \times 10^{13}$ photons $/ \mathrm{cm}^{2}$ $\left(30 \mu \mathrm{J} / \mathrm{cm}^{2}\right)$ to a maximum of $4.4 \times 10^{15}$ photons $/ \mathrm{cm}^{2}\left(2260 \mu \mathrm{J} / \mathrm{cm}^{2}\right)$ per pulse, using a variable neutral density filter. We worked mainly with $530 \mathrm{~nm}$ excitation, but recorded also some spectra at $475 \mathrm{~nm}$ and $650 \mathrm{~nm}$ excitation wavelengths.

The THz probe radiation was generated in a $1 \mathrm{~mm}$ thick zinc telluride ( $\mathrm{ZnTe}$ ) crystal (optical rectification). Using two gold-coated off-axis parabolic mirrors, the $\mathrm{THz}$ beam was then focused on the sample. The THz beam propagated in a box purged with dry nitrogen. Another pair of parabolic mirrors was used to collect the THz pulses transmitted through the sample and to focus them onto a second ZnTe crystal for the detection. A gating beam at $800 \mathrm{~nm}$ was also going through this ZnTe detection crystal. The THz radiation induced a birefringence inside the crystal, which changed the polarization of the initially linearly polarized gating beam, when there was temporal coincidence (electro-optic 
sampling). A Wollaston prism separated the horizontal and the vertical components of the elliptical polarized light [17]. Finally the intensity of the two components was detected by a pair of balanced photodiodes, which monitored the change in polarization induced by the $\mathrm{THz}$ radiation. By varying the time delay between the gating and the $\mathrm{THz}$ beams (using a computerized delay stage), the entire $\mathrm{THz}$ electric field could be mapped out.

\section{RESULTS AND DISCUSSION}

\subsection{Neat PBDTTPD}

The normalized transient THz dynamics for neat and blended PBDTTPD are shown in Figure 2, for $530 \mathrm{~nm}$ excitation and at high fluence $\left(1646 \mu \mathrm{J} / \mathrm{cm}^{2}\right)$. The data was obtained by taking the difference between the pumped and un-pumped transmitted $\mathrm{THz}$ signal $(\Delta \mathrm{T})$ divided by the maximum intensity of the un-pumped $\mathrm{THz}$ pulse $(\mathrm{T})$.

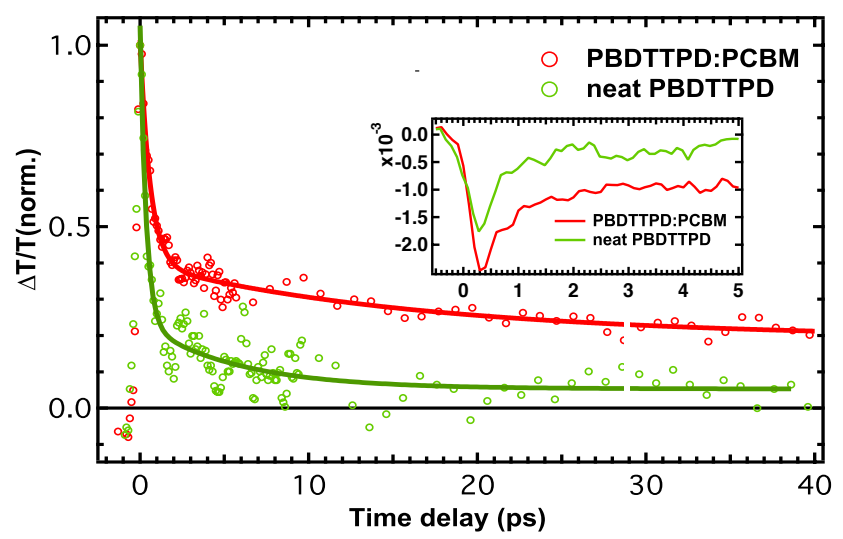

Figure 2 Transient THz dynamics of neat PBDTTPD and PBDTTPD:PCBM for high fluence of $4.4 \cdot 10^{15}$ photons $/ \mathrm{cm}^{2}(1646$ $\mu \mathrm{J} / \mathrm{cm}^{2}$ ), normalized and not normalized (inset).

Despite the high fluence used for these experiments, the difference in the decay of the transient $\mathrm{THz}$ signal between the neat and the blended sample is already very clear: in the neat film after a prompt signal rise, a very fast decay (within a few ps) is observed. In this case, mainly excitons contribute to the (weaker) THz signal, although we cannot exclude the presence of some charges. Although excitons, unlike charges, are not resonant with $\mathrm{THz}$ radiation, their polarizability can affect the transmitted $\mathrm{THz}$ probe. Fast exciton-exciton annihilation phenomena are prevailing at the high fluence, explaining why the generated excitons have already mostly recombined after 40 ps (there is only a very weak longerlived offset). In contrast, the natural lifetime of excitons in PBDTTPD is $150 \mathrm{ps}$, as seen by TA spectroscopy [18].

On the other hand, in the blend even at high fluence, it is clear that part of the transient THz signal survives also after 40 ps, most likely due to the generation of charges in the blend. The fast (few picosecond) decay dynamics in this case is mainly due to bimolecular recombination of the charges, although exciton-exciton and exciton-charge annihilation might also contribute. Figure 2 shows in the inset the not-normalized transient $\mathrm{THz}$ dynamics for the neat and blended samples: surprisingly the amplitude for the neat sample is comparable to the one for the PBDTTPD:PCBM blend. Since the amplitude of the $\mathrm{THz}$ signal should be proportional to the population of resonant photoexcited charges, it would be expected that the amplitude in the blend, where charge generation is enhanced, is much higher. This is probably due to the strong artefacts induced by the annihilation phenomena present at this high fluence, which are obscuring the real mechanism of free charges generation.

The transient THz dynamics of the neat PBDTTPD film for lower fluencies $\left(90 \mu \mathrm{J} / \mathrm{cm}^{2}\right.$ and $\left.30 \mu \mathrm{J} / \mathrm{cm}^{2}\right)$ are shown in Figure 3. We note that they were recorded with a different film of PBDTTPD, which might lead to some differences compared to the data in Figure 2. 


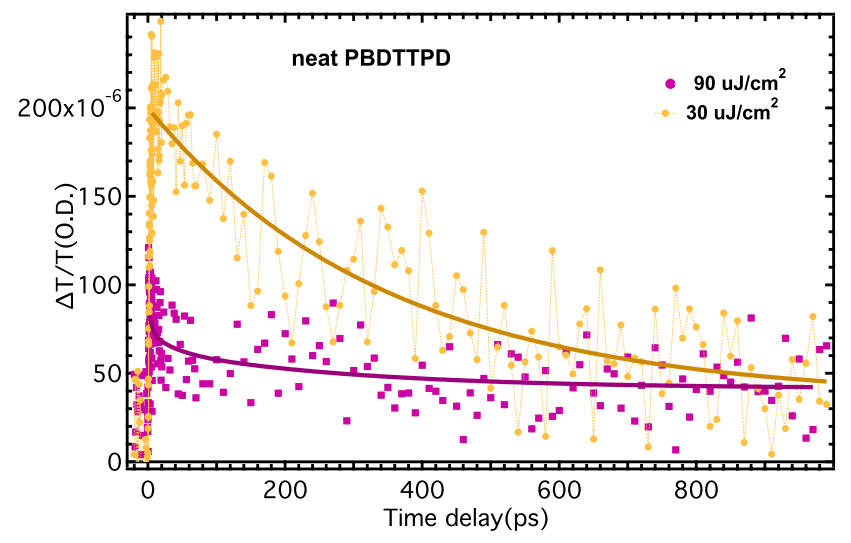

Figure 3 Transient THz dynamics of neat PBDTTPD samples excited at $530 \mathrm{~nm}$ with $30 \mu \mathrm{J} / \mathrm{cm}^{2}$ and $90 \mu \mathrm{J} / \mathrm{cm}^{2}$

The dynamics in Figure 3 for the low $30 \mu \mathrm{J} / \mathrm{cm}^{2}$ excitation fluence has been analyzed using a bi-exponential function with time constants of $6 \mathrm{ps}$ (weak) and $150 \mathrm{ps}$ (predominant). We found similar time constants using TA spectroscopy to study the dynamics of excitons in neat PBDTTPD films [5]. This supports that the $150 \mathrm{ps}$ component in the THz data indeed describes the decay of relaxed excitons to the ground state. Also, Laquai et al found a time constant of $200 \mathrm{ps}$ with photoluminescence measurements in PBDTTPD films, which was attributed to the lifetime of intramolecular excited state excitons [3]. Hence the THz dynamics in Figure 3 are predominantly describing the recombination of exciton species (in the absence of annihilation artefacts), confirming that the bound excitons affect the transmitted $\mathrm{THz}$ probe beam and that the dynamics are not only due to photogenerated charges. The confirmation of this picture comes from the photoconductivity spectra recorded at $0.5 \mathrm{ps}$ and $800 \mathrm{ps}$ time delays, shown below.

The transient $\mathrm{THz}$ dynamics for the $90 \mu \mathrm{J} / \mathrm{cm}^{2}$ fluence present a different behavior. Surprisingly, the data in Figure 3 shows a higher amplitude for the lower fluence $\left(30 \mu \mathrm{J} / \mathrm{cm}^{2}\right)$ in comparison to the dynamic at $90 \mu \mathrm{J} / \mathrm{cm}^{2}$. The latter has been analyzed using a biexponential function with a long component of $1 \mathrm{~ns}$. At this fluence, annihilation phenomena have a more important contribution [4]. Banerji et al. have shown that for high fluencies, the dynamics of the neat films can be described with additional fast components of $0.35 \mathrm{ps}$ and $22 \mathrm{ps}$. Hence, it is probable that the lower amplitude for the $90 \mu \mathrm{J} / \mathrm{cm}^{2}$ fluence can be explained by annihilation processes that are faster than the time resolution of the experiment $(\approx 300 \mathrm{fs})$. Intramolecular excitons thus become "invisible" in the THz data due to ultrafast annihilation. We tentatively suggest that the long $1 \mathrm{~ns}$ component is due to more delocalized intermolecular excitons, which are less sensitive to annihilation. Laquai et al. have recently shown the existence of such long-lived species in PBDTTPD [3].

Photoconductivity spectra in the $\mathrm{THz}$ range have been widely used to study inorganic semiconductors (e.g. GaAs),[19] and supraconductors. The typical spectral shape found for inorganic materials and for highly doped materials [20] presents a positive real part with a maximum at zero frequency and a positive imaginary part. This shape corresponds to a Drude like model, which describes a material characterized by free charges, as discussed in Chapter 2. A completely different shape has been found in $\mathrm{THz}$ spectra of neat conjugated polymers and polymer:fullerene blends that have been recently studied $[12,21,22]$. In fact, the maximum of the positive real part is no longer at zero frequency and the imaginary part is negative, displaying a minimum value at a non-zero frequency. This behaviour is physically corresponding to a picture in which the charges behave differently compared to inorganic semiconductors. This can come from the presence of bound charges (restoring force) or from backscattered charges.

The commonly reported $\mathrm{THz}$ photoconductivity signature for neat polymers in relevant literature presents a monotonical increase with the $\mathrm{THz}$ frequency in the positive real and negative imaginary part. This behavior has been found specifically for semi-crystalline polymers such as P3HT between 0.2 and $2 \mathrm{THz}$ [23] and also for MEH-PPV between 0.2 and $1.2 \mathrm{THz}$ [15]. The authors corroborate that this behavior is characteristic of a dispersive transport in a disordered medium. As the polymers exhibit nanoscale disorder, the model used to describe the spectra is the Drude-Smith model, which accounts for strong carrier localization. It seems that the species observed in P3HT and MEH-PPV are weakly bound excitons. Also Sundstroem et al. observe weakly coulombically bound species in neat APFO polymer. 
Figure 4 shows the photoconductivity spectra for neat PBDTTPD film recorded at time delays of 0.5 ps and 800 ps, with a high fluence of $4.4 \cdot 10^{15}$ photons $/ \mathrm{cm}^{2}$ at an excitation wavelength of $530 \mathrm{~nm}$.
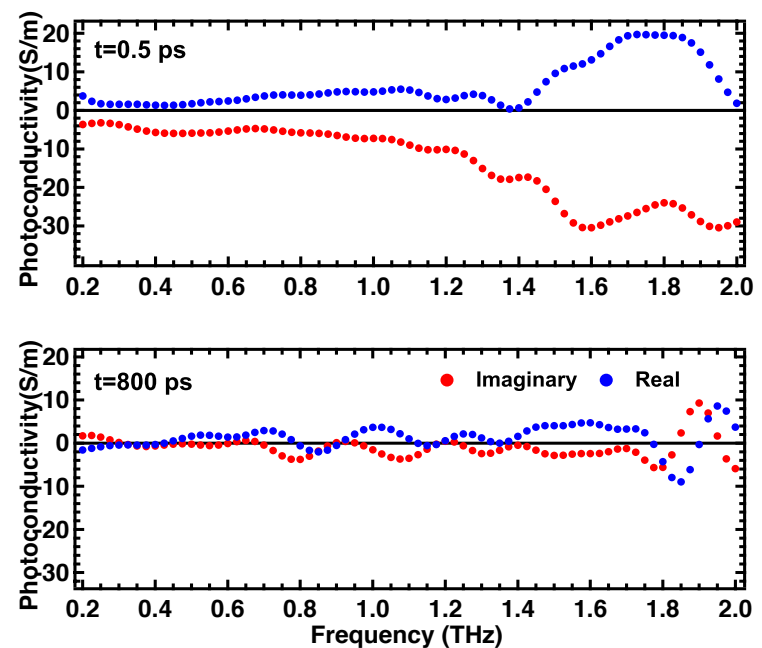

Figure $4 \mathrm{THz}$ photoconductivity spectra of neat PBDTTPD film recorded at time delays of $0.5 \mathrm{ps}$ and $800 \mathrm{ps}$ using a fluence of $4.4 \cdot 10^{15}$ photons $/ \mathrm{cm}^{2}$ at an excitation wavelength of $530 \mathrm{~nm}$.

Comparing the spectra at $0.5 \mathrm{ps}$ and $800 \mathrm{ps}$, it is clear that practically all the excitons and charges have recombined after $800 \mathrm{ps}$, as has been shown already in the transient $\mathrm{THz}$ dynamics for high fluencies reported in Figure 2 . The microscopic origin of the photoconductivity signature in organic materials is a topic of some debate. A close look at the 0.5 ps spectrum reveals that the real part of the photoconductivity is almost zero for low $\mathrm{THz}$ frequencies and becomes slightly higher for higher frequencies, reaching a maximum at $1.7 \mathrm{THz}$. The negative imaginary part is more important than the positive real part, as it has a larger amplitude. It has a minimum at a $\mathrm{THz}$ frequency slightly lower than the maximum of the real part. This asymmetrical behaviour between the real part and the imaginary part seems to describe mostly bound species. Among all the theoretical models [24], the presence of a maximum in the real part and a minimum in the imaginary part corresponds to a photophysical situation in which there is the presence of a restoring force. The latter can come from back-scattering from the end of a polymer segment (Drude-Smith model) or from a torsional disorder [25]. In the Drude-Smith model, the negative imaginary part should become zero in correspondence with the maximum of the positive real part. Yet this is not the case, so that we tentatively suggest that the observed shape of the photoconductivity spectra is closer to an Oscillator-Lorenzian model.

The presence of bound species in the neat PBDTTPD sample is further confirmed if we consider the transmitted THz electric field $0.5 \mathrm{ps}$ after photoexcitation shown in Figure 5. This is the difference $(\Delta \mathrm{E})$ between $\mathrm{E}_{\text {pumped }}(\mathrm{THz}$ electric field in the presence of the excitation pump) and $\mathrm{E}_{\text {unpumped }}\left(\mathrm{THz}\right.$ electric field without the pump). The $\mathrm{E}_{\text {unpumped }}$ field is shown as a reference. For samples with an important presence of photogenerated free charges, $\Delta \mathrm{E}$ resembles the mirror image of the unpumped field, indicating absorption of the $\mathrm{THz}$ radiation by resonant charges. In contrast, the $\Delta \mathrm{E}$ signal for neat PBDTTPD film (red curve in Figure 5a)) is reproducing the same shape as the unpumped field with the maximum shifted to the right. This is due to the presence of bound species in the neat sample, which do not absorb the $\mathrm{THz}$ radiation (as free charges do) but shift it temporally. Hence the field appears to be only delayed in time with respect to the unpumped radiation. This physical behavior is a clear signature of excitons in the neat phase of PBDTTPD. Hence, the shifted $\mathrm{THz}$ electric field gives some information about the fact that the species we detect have a large polarizability and in this situation also an important restoring force. The Fourier transformation of $\mathrm{E}_{\text {unpumped }}$ and $\Delta \mathrm{E}$ shown in Figure $5 b)$ gives information about the $\mathrm{THz}$ range in which it is possible to obtain useful information and to extract the photoconductivity spectra. 

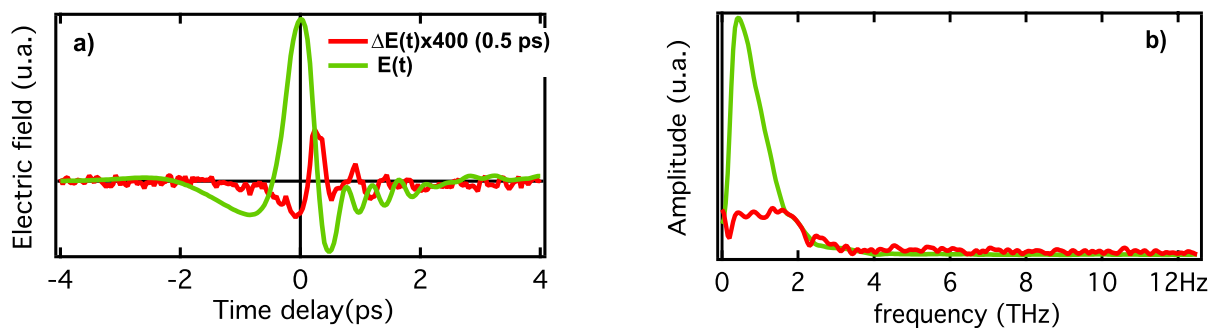

Figure 5 a) Transmitted unpumped THz electric field (green) for neat PBDTTPD film and difference between the pumped $\mathrm{THz}$ field and the unpumped $\mathrm{THz}$ field $\left(\Delta \mathrm{E}=\mathrm{E}_{\text {pumped }}-\mathrm{E}_{\text {unpumped}}\right.$, red), $0.5 \mathrm{ps}$ after excitation at $530 \mathrm{~nm}$ with a fluence of $4.4 \cdot 10^{15}$ photons $/ \mathrm{cm}^{2}$, multiplied by a factor 400 to allow the comparison. b) Fourier-transformed THz spectrum (frequency domain) of the $\mathrm{THz}$ fields shown in panel a).

To confirm that the information in Figure 5 is not only related to high fluence, Figure 6 shows the transmitted $\mathrm{THz}$ electric field (unpumped and $\Delta \mathrm{E}, 20 \mathrm{ps}$ after excitation) for a lower fluence of $90 \mu \mathrm{J} / \mathrm{cm}^{2}$ (the related dynamics are shown in Figure 3). Also in this case, the shift of the $\mathrm{THz}$ field corresponds to a situation where the generated species are bound, as expected for excitons in neat PBDTTPD film.

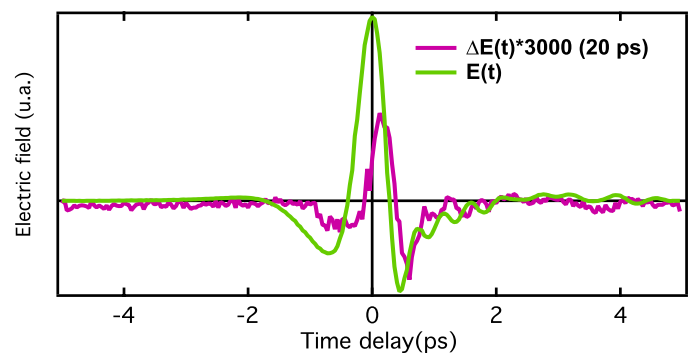

Figure 6 Transmitted THz electric field for neat PBDTTPD film, for the unpumped case and $\Delta \mathrm{E}=\mathrm{E}_{\text {pumped }}-\mathrm{E}_{\text {unpumped }}, 20 \mathrm{ps}$ after excitation at $530 \mathrm{~nm}$ with $90 \mu \mathrm{J} / \mathrm{cm}^{2}$ fluence (corresponding dynamics are shown in dark pink in Figure 3).

\subsection{PBDTTPD:PCBM Blend}

The normalized transient THz dynamics for the PBDTTPD:PCBM blend are shown in Figure 7 for $530 \mathrm{~nm}$ excitation at various fluencies. The analysis was done using three-exponential functions

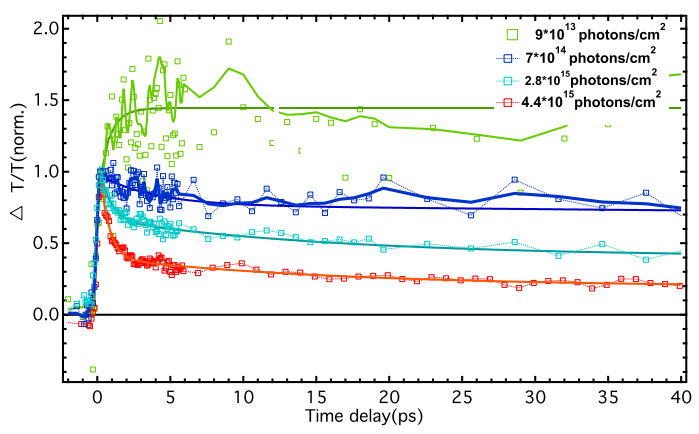

Figure 7: Transient THz dynamics for PBDTTPD:PCBM, with varying excitation fluence at $530 \mathrm{~nm}: 9 \cdot 10^{13} \mathrm{photons} / \mathrm{cm}^{2}(30$ $\left.\mu \mathrm{J} / \mathrm{cm}^{2}\right), 7 \cdot 10^{14}$ photons $/ \mathrm{cm}^{2}\left(264 \mu \mathrm{J} / \mathrm{cm}^{2}\right), 2.8 \cdot 10^{15}$ photons $/ \mathrm{cm}^{2}\left(1055 \mu \mathrm{J} / \mathrm{cm}^{2}\right)$, and $4.4 \cdot 10^{15} \mathrm{photons} / \mathrm{cm}^{2}\left(1646 \mu \mathrm{J} / \mathrm{cm}^{2}\right)$. The data have been normalized at $0.2 \mathrm{ps}$. 
The dynamics for the highest intensity shows an instantaneous rise, a fast decay and then a slower decay of $1000 \mathrm{ps,}$, in agreement with the discussion of Figure 2. Since for the high excitation intensity, we have a higher density of photogenerated excitons and charges, the exciton annihilation and bimolecular charge pair recombination phenomena are prevailing, which causes the very fast decay times. This agrees with the results obtained with TA spectroscopy by Banerji et al. [4]. It is probable that we missed part of the ultrafast decay, which occurs faster than the time resolution. By decreasing the fluence, the amplitude of the fast decay in the $\mathrm{THz}$ dynamics becomes less pronounced. When the fluence is reduced by two orders of magnitude, we observe instead a 1 ps rise in the dynamics (Figure 7). This originates from the generation of charges directly by exciton dissociation. The 1 ps rise is in agreement with the delayed 1 ps CS observed in the TA experiments (visible pump and probe, section 5.4.2) [18]. Those experiments showed that CS in PBDTTPD:PCBM is partly prompt $(<100 \mathrm{fs})$ and partly delayed $(1 \mathrm{ps})$, for excitons generated in neat polymer domains that need to diffuse to an interface. To our knowledge, we report here the first observation of delayed charge generation in polymer:fullerene blends by $\mathrm{THz}$ spectroscopy. This outlines the importance of using low excitation intensity in $\mathrm{THz}$ experiments in order to observe the real dynamics of charge generation.

The local mobility of photogenerated charges could be estimated from the non-normalized amplitude of the $\mathrm{THz}$ dynamics. In fact, it is possible to extract the product of the mobility $(\mu)$ and of the charge generation efficiency $(\phi)$.from the initial (picosecond) $\Delta \mathrm{T} / \mathrm{T}$ amplitude (not normalized), using the following equation:

$$
\mu \phi=\frac{\Delta \sigma_{0}}{e n_{0}^{\text {photon }}}
$$

$n_{0}^{\text {photon }}=\left(1-e^{-\alpha d}\right) \mathrm{I}_{0} / \mathrm{d}$ is the photoexcited carrier density, extracted from the absorption coefficient $\alpha$, the number of incident photons $\mathrm{I}_{0}$ per unit of area, and the film thickness $\mathrm{d}$. e is the electronic charge and $\Delta \sigma_{0}$ is the photoconductivity, i.e. the change in sample conductivity extracted from the change in $\mathrm{THz}$ transmission $\Delta \mathrm{T} / \mathrm{T}$ [26]:

$$
\Delta \sigma_{0}=\frac{1+n}{Z_{0} d}\left(\frac{1}{1+\frac{\Delta \mathrm{T}}{\mathrm{T}}}-1\right)
$$

$Z_{0}$ is the impedance of free space, $d$ is the thickness of the sample and $n$ is the quartz substrate refractive index.[27]

The values of the local mobility extracted from the photoconductivity measurements (in Figure 7) for PBDTTPD:PCBM are shown in Table 1. These values are of the same order of magnitude of as the ones found by Parkinson et al. in P3HT:PCBM [26], who reported a value of $\mu \phi=0.11 \mathrm{~cm}^{2} / \mathrm{Vs}$ at a fluence of $500 \mu \mathrm{J} / \mathrm{cm}^{2}$ (after a fitting procedure of photoconductivity as a function of fluence), and by Turchinovih et al. in PTB7:PCBM [12], who found a value of $\mu \phi=0.3 \mathrm{~cm}^{2} / \mathrm{Vs}, 0.3 \mathrm{ps}$ after excitation at $530 \mu \mathrm{J} / \mathrm{cm}^{2}$ (extracted from the Drude-Smith model). We note that the $\mu \phi$ product gives in our case a lower-limit value of the local mobility, since the charge generation efficiency at the initial time delay is $<1$ due to delayed CS. The decrease of $\mu \phi$ at higher fluencies might be related to a further decrease of $\phi$ caused by annihilation faster than the time resolution of the experiment. However, the precise connection between local mobility and fluence needs further investigation and a broader range of fluencies.

\begin{tabular}{cc}
\hline $\begin{array}{c}\text { Fluence } \\
\left(\mu \mathbf{J} / \mathbf{c m}^{2}\right)\end{array}$ & $\begin{array}{c}\text { Local Mobility } \\
\left(\mathbf{c m}^{2} / \mathrm{Vs}\right)\end{array}$ \\
$\mathbf{2 6 4}$ & 0.066 \\
$\mathbf{1 2 1}$ & 0.11 \\
$\mathbf{5 5}$ & 0.14 \\
$\mathbf{3 0}$ & 0.17 \\
\hline
\end{tabular}

Table 1 Local mobility multiplied by the efficiency of charge generation $(\mu \phi)$ for different fluencies for PBDTTPD:PCBM. 
Long-range mobility measurements have been performed by McGehee et al. on the same polymer:fullerene blend using current-voltage characteristics. They found values orders of magnitude smaller compared to the local mobility that we report using non-contact THz spectroscopy (the long-range hole mobility is of the order of $10^{-4} \mathrm{~cm}^{2} / \mathrm{Vs}$ for the neat and $10^{-5} \mathrm{~cm}^{2} / \mathrm{Vs}$ in the blended sample). The physical meaning of the local mobility probed by THz spectroscopy has been recently explained by McGehee [28]. It is more related to the frequency of attempts for an electron-hole pair to split (the intrinsic hopping rate) and to charge motion on the very short length scale (without grain boundaries). The local mobility has also been found using a non-contact probe in the microwave range (time-resolved microwave conductivity, TRMC) [29]. Their values are slightly lower in relation to the ones found with the THz method. Excitation fluence might have affected the local mobility in the microwave field for neat P3HT.

The photoconductivity spectra shown in Figure 8 for the PBDTTPD:PCBM blend, excited at $530 \mathrm{~nm}$ at a fluence of $4.4 \cdot 10^{15}$ photons $/ \mathrm{cm}^{2}$ per pulse confirm that the generated species are charges. In fact the photoconductivity spectra, as discussed above, allow to distinguish bound species from charges. The real and imaginary part of the photoconductivity has been calculated from the amplitude and the phase of the transmitted $\mathrm{THz}$ electric field with respect to a reference quartz plate, at two fixed pump-probe delay times. The spectra obtained at $0.5 \mathrm{ps}$ and $800 \mathrm{ps}$ are shown in Figure 8.
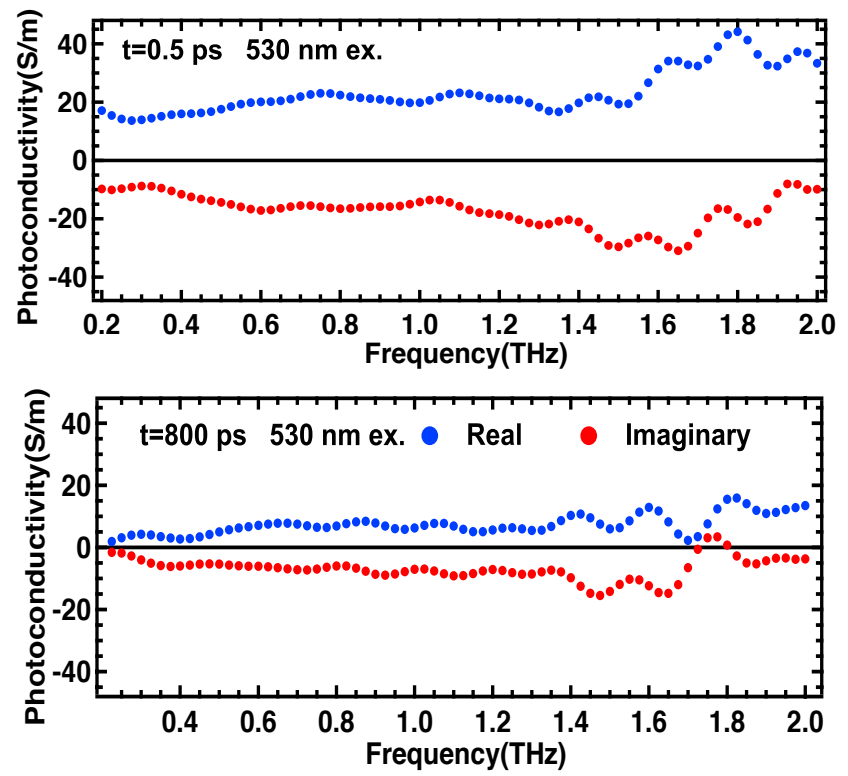

Figure 8: Photoconductivity spectra for PBDTTPD:PCBM at a time delay of 0.5 ps and 800 ps following excitation at 530 nm with $4.4 \cdot 10^{15}$ photons $/ \mathrm{cm}^{2}$ per pulse.

The situation is quite different from the spectra described for the neat polymer (Figure 4). In particular, the amplitude of the real part is more important than the corresponding real part in the neat sample, especially at the lower $\mathrm{THz}$ frequencies. This is a clear signature of the presence of free charges (polarons) while the presence of the negative part is describing a localization of charges. The latter could come from some backscattering effect (caused by disorder effects) or due to charges that are not completely free (in contrast to an inorganic material), which may be confined polarons. Hence, the photoexcited species observed at $0.5 \mathrm{ps}$ still have some characteristics of a bound system, possibly also due to the delayed presence of excitons or to the formation of a charge transfer (CT) state.

At $0.5 \mathrm{ps}$, it is possible to observe a positive peak for the real part of the photoconductivity around $1.7 \mathrm{THz}$, and a negative peak for the imaginary part around $1.5 \mathrm{THz}$. The spectral shape with a maximum and a minimum shown in Figure 8 has never been observed for polymer:fullerne blends in the range between $0.2 \mathrm{THz}$ and $2 \mathrm{THz}$. It needs further confirmation and investigation. Turchinovich et al. found in the same $\mathrm{THz}$ range for an amorphous polymer blend (PTB7:PCBM) a spectral shape with the real and imaginary part increasing in amplitude at higher frequency. They suggest that this shape originates from a model where the mobile charges are hindered by a potential well created in a polaron pair. The photophysical model used to describe this situation is the Drude-Smith model. They found also that the mobility decreases at higher excitation density with increasing carrier-carrier interaction [12]. 
In Figure 8, it is possible to observe a decrease in the amplitude of the $\mathrm{THz}$ photoconductivity going from 0.5 ps to 800 ps, since less charge density survived until 800 ps (annihilation and recombination effects, see Figure 7). At 800 ps, there is evidence that the charges are the same kind of species as seen at $0.5 \mathrm{ps}$. This is also confirmed by looking at Figure 9 , which shows the ratio between the real and the imaginary part of the photoconductivity at $0.5 \mathrm{ps}$ and $800 \mathrm{ps}$. It is clear that the ratio has for both time delays almost the same value, indicating that the species detected are similar. The important imaginary part indicates that bound species are somewhat present at long time delays, which could be a contribution of (delocalized interchain) excitons that decay more slowly, or of bound charge pairs, but is most probably related to the polaronic nature of relatively free charges in disordered polymers.

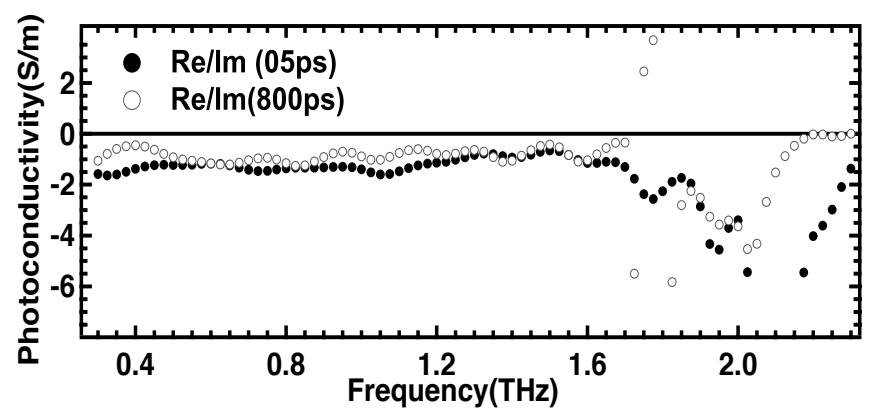

Figure 9: Ratio between the real and the imaginary part of the photoconductivity spectra of PBDTTPD:PCBM shown in Figure 8 , at 0.5 ps and 800 ps.

Figure 10 illustrates the photo-modulation of the THz electric field $\left(\Delta \mathrm{E}=\mathrm{E}_{\mathrm{pumped}}-\mathrm{E}_{\text {unpumped }}\right)$ at $0.5 \mathrm{ps}$ and $800 \mathrm{ps}$, with respect to the transmitted unpumped THz electric field for the PBDTTPD:PCBM blend. In contrast to the photomodulated electric field for neat PBDTTPD polymer shown in Figure 5, it is clear that the $\Delta \mathrm{E}$ electric fields at both delay times mirror the unpumped electric field, and are only very slightly shifted to the left side by $0.1 \mathrm{ps}$ (the origin of the shift is unclear and possibly within the noise). This characteristic behavior clearly corresponds to a photophysical picture where the $\mathrm{THz}$ field is absorbed by the photo-generated charges in the blended sample, and where the restoring force, which is typical of a strongly polarizable exciton species, is absent. The different amplitude between the $\Delta \mathrm{E}$ field at 0.5 ps and the field at $800 \mathrm{ps}$ is coming from the bimolecular recombination phenomena at high fluence. Finally, it is possible to consider the photo-modulation of the electric field at $0.5 \mathrm{ps}$ and $800 \mathrm{ps}$ displayed in Figure 10 as the $\mathrm{THz}$ signature of the charges (polarons). A similar signature at $2 \mathrm{ps}$ has been found by Turchinovich et al. [12] on another amorphous polymer (PTB7) blended with PCBM and also by J. Elligson et al. on P3HT:PCBM [30].

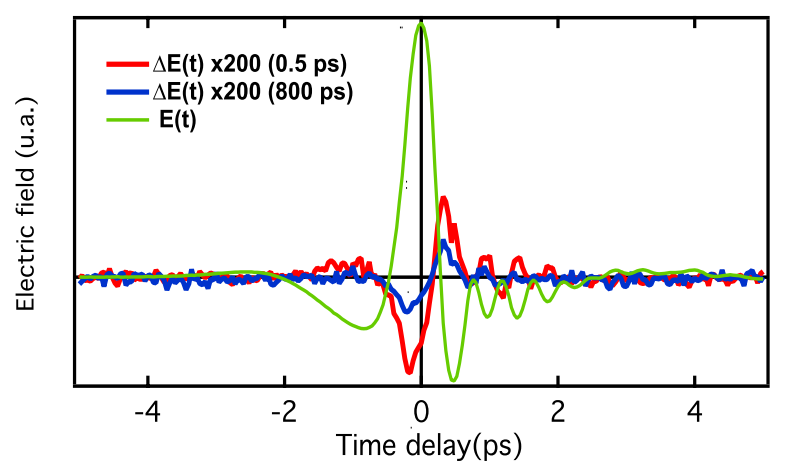

Figure 10 Transmitted THz electric field recorded without pump (green), and photo-modulated electric field $\left(\Delta \mathrm{E}=\mathrm{E}_{\text {pumped }}\right.$ $\mathrm{E}_{\text {unpumped }}$ ) for PBDTTPD:PCBM at time delays of $0.5 \mathrm{ps}$ and $800 \mathrm{ps}$ following excitation at $530 \mathrm{~nm}$ with $4.4 \cdot 10^{15}$ photons $/ \mathrm{cm}^{2}$ per pulse. 
The above interpretation can be confirmed for a measurement at low fluence $\left(9 \cdot 10^{13}\right.$ photons $\left./ \mathrm{cm}^{2}\right)$, as shown in Figure 11. In fact, the modulated electric fields at $0.5 \mathrm{ps}$ and at $800 \mathrm{ps}$ have the same shape as the ones shown in Figure 10 for the highest fluence, and mirror the unpumped field. The amplitude at the two time delays is nevertheless now similar. This is due to the fact that at this fluence, the generated charges do not recombine on the $1 \mathrm{~ns}$ time scale because the bimolecular recombination phenomena have been eliminated. They do not undergo a significant decrease in local mobility, either, as this would also affect the THz signal. Figure 11 is decisively showing that the charges at $0.5 \mathrm{ps}$ and $800 \mathrm{ps}$ are the same kind of species. Specifically the detected species are related to free charges (polarons), since the characteristic mirrored disposition of the modulated field with respect to the unpumped field clearly describes a situation where the energy contained in the $\mathrm{THz}$ electric field is absorbed and not delayed by the generated charges (i.e. the generated charges are relatively free to react to the probe $\mathrm{THz}$ field). Hence it is possible to conclude that the process of free charge generation in the blend seems to happen following a direct, instantaneous pathway.

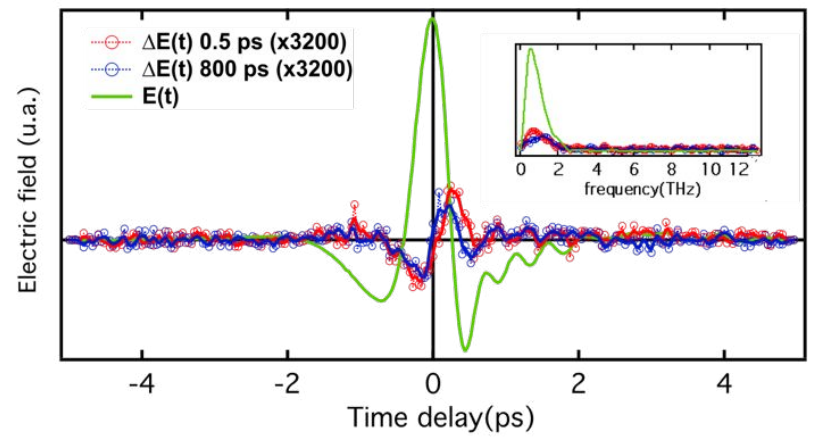

Figure 11 Transmitted THz electric field recorded without pump (green), and photo-modulated electric field $\left(\Delta \mathrm{E}=\mathrm{E}_{\text {pumped }}-\right.$ $\mathrm{E}_{\text {unpumped }}$ ) for PBDTTPD:PCBM at time delays of $0.5 \mathrm{ps}$ and $800 \mathrm{ps}$ following excitation at $530 \mathrm{~nm}$ with $9 \cdot 10^{13}$ photons $/ \mathrm{cm}^{2}$ $\left(30 \mu \mathrm{J} / \mathrm{cm}^{2}\right)$ per pulse.

The connection between the shape of the photo-modulated $\mathrm{THz}$ field and the presence of charges can be further confirmed if we study the blended sample after performing an above-gap excitation at $475 \mathrm{~nm}(2.6 \mathrm{eV})$, shown in Figure 12 for a time delay of $0.5 \mathrm{ps}$ (the energy gap of the PBDTTPD is $1.8 \mathrm{eV}$ ). Indeed the THz field $\Delta \mathrm{E}(\mathrm{t}, \Delta \mathrm{t}=0.5 \mathrm{ps})$ shows all the characteristics connected with the presence of charges: it mirrors the unpumped $\mathrm{THz}$ field and it is not shifted with respect to it, indicating absorption of the $\mathrm{THz}$ probe.

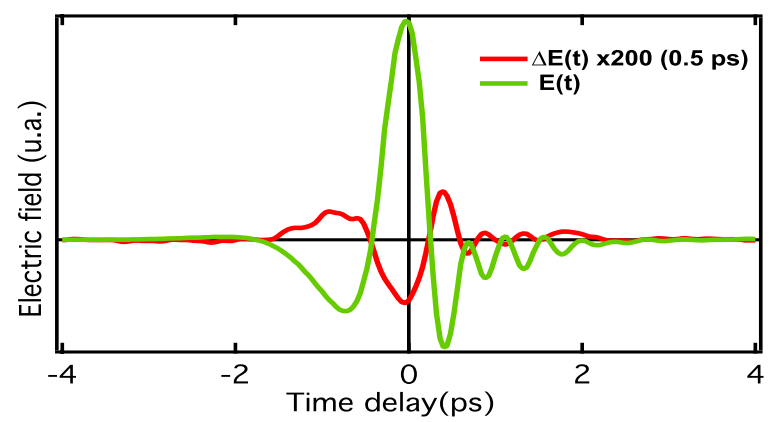

Figure 12 Transmitted THz electric field recorded without pump (green), and photo-modulated electric field $\Delta \mathrm{E}(\mathrm{t}, \Delta \mathrm{t}=0.5 \mathrm{ps})$ for PBDTTPD:PCBM at a time delay of $0.5 \mathrm{ps}$ after excitation at $475 \mathrm{~nm}$ with $4.4 \cdot 10^{15}$ photons $/ \mathrm{cm}^{2}\left(30 \mu \mathrm{J} / \mathrm{cm}^{2}\right)$ per pulse. 
The photoconductivity spectra for the $475 \mathrm{~nm}$ excitation wavelength are shown in Figure 13 and are compared with the ones for the $530 \mathrm{~nm}$ excitation wavelength. It is obvious that the amplitude of the real part of the photoconductivity is more important than the imaginary part, which is almost zero at $475 \mathrm{~nm}$. Also, the shape of the real part is different in relation to the one at $530 \mathrm{~nm}$ : it increases linearly with the frequency and it does not have any peak. This behavior is more similar to the signature of the real part of the photoconductivity for P3HT:PCBM. The important amplitude of the real part, and quasi absence of the imaginary part, correspond to a picture where the charges are less bound than with excitation at $530 \mathrm{~nm}$. Specifically, the increase of the real part with frequency could describe a situation where the charges are free to respond to the THz probe, in contrast to the real part at $530 \mathrm{~nm}$ excitation wavelength. This suggests that excess excitation energy can have some effect on the nature of photo-generated charges, but needs further investigation.

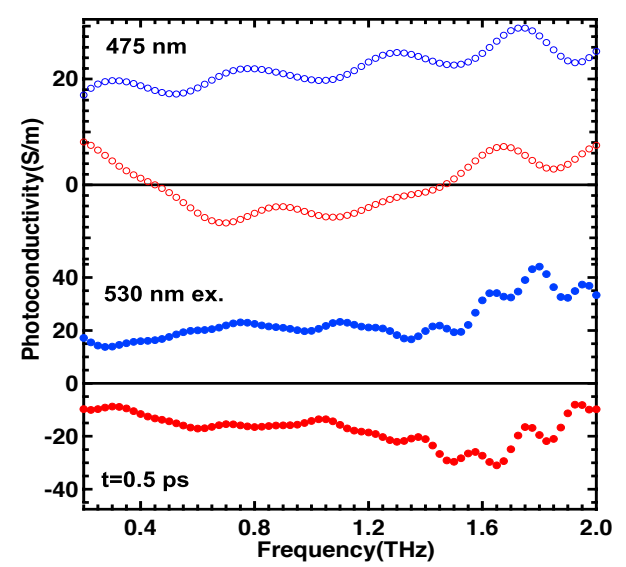

Figure 13 Comparison between the frequency dependence of the real part (blue circles) and the imaginary part (red circles) of the THz photoconductivity in PBDTTPD:PCBM measured for $530 \mathrm{~nm}$ excitation wavelength (full circles) and $475 \mathrm{~nm}$ excitation wavelength (empty circles) at a time delay of $0.5 \mathrm{ps}$ for $4.4 \cdot 10^{15}$ photons $/ \mathrm{cm}^{2}$.

\section{CONCLUSION}

We have investigated the photophysics in neat PBDTTPD films and PBDTTPD:PCBM blends using terahertz timedomain spectroscopy with excitation at $530 \mathrm{~nm}$ or $475 \mathrm{~nm}$. THz measurements are sensitive to detect charges and to identify their nature (bound or free). We were able to identify a THz signature for bound excitons in neat PBDTTPD films, pointing to important delocalization in those excitons. Then, we investigated the nature and local mobility (orders of magnitude higher than bulk mobility) of charges in the PBDTTPPD:PCBM blend as a function of excitation wavelength, fluence and pump-probe time delay. Low fluencies, which allowed the removal of bimolecular recombination phenomena, were needed to evidence the charge generation dynamics and the accurate values of local charge mobility. The charge generation in the blended sample had a delayed component originating from excitons created in neat polymer domains which, thanks to delocalization, could reach the PCBM interface and dissociate to charges on a time scale of $1 \mathrm{ps}$. From the THz measurements it was possible to confirm that the excitons split directly into relatively free charges within the time resolution of the experiment. 


\section{REFERENCES}

[1] C. Cabanetos, A. El Labban, J. A. Bartelt et al., "Linear Side Chains in Benzo[1,2-b:4,5-b']dithiopheneThieno[3,4-c]pyrrole-4,6-dione Polymers Direct Self-Assembly and Solar Cell Performance,” J. Am. Chem. Soc., 135, 4656-4659 (2013).

[2] J. A. Bartelt, Z. M. Beiley, E. T. Hoke et al., "The importance of fullerene percolation in the mixed regions of polymer-fullerene bulk heterojunction solar cells," Adv. Energy Mater., 3, 364-374 (2013).

[3] C. Dyer-Smith, Howard, I. A., Cabanetos, C., Labban, A. E., Beaujuge, P. M. and Laquai, F., "Interplay between side chain pattern, polymer aggregation, and charge carrier dynamics in PBDTTPD:PCBM bulkheterojunction solar cells," Adv. Energy Mater., 5, 1401778 (2015).

[4] A. A. Paraecattil, and N. Banerji, "Charge separation pathways in a highly efficient polymer: fullerene solar cell material," J. Am. Chem. Soc., 136, 1472-1482 (2014).

[5] A. A. Paraecattil, S. Beaupre, M. Leclerc et al., "Intensity dependent femtosecond dynamics in a PBDTTPDbased solar cell material," J. Phys. Chem. Lett., 3, 2952-2958 (2012).

[6] O. Esenturk, J. S. Melinger, and E. J. Heilweil, "Terahertz mobility measurements on poly-3-hexylthiophene films. Device comparison, molecular weight, and film processing effects," J. Appl. Phys., 103, 023102 (2008).

[7] F. Wang, J. Shan, M. A. Islam et al., "Exciton polarizability in semiconductor nanocrystals," Nat. Mater., 5, 861-864 (2006).

[8] R. Mauer, M. Kastler, and F. Laquai, "The Impact of Polymer Regioregularity on Charge Transport and Efficiency of P3HT:PCBM Photovoltaic Devices,” Adv. Funct. Mater., 20, 2085-2092 (2010).

[9] O. Esenturk, R. J. Kline, D. M. Delongchamp et al., "Conjugation Effects on Carrier Mobilities of Polythiophenes Probed by Time-Resolved Terahertz Spectroscopy,” J. Phys. Chem. C, 112, 10587-10590 (2008).

[10] P. D. Cunningham, and L. M. Hayden, "Carrier Dynamics Resulting from Above and Below Gap Excitation of P3HT and P3HT/PCBM Investigated by Optical-Pump Terahertz-Probe Spectroscopy,” J. Phys. Chem. C, 112, 7928-7935 (2008).

[11] C. S. Ponseca, A. Yartsev, E. Wang et al., "Ultrafast Terahertz Photoconductivity of Bulk Heterojunction Materials Reveals High Carrier Mobility up to Nanosecond Time Scale,” J. Am. Chem. Soc., 134, 11836-11839 (2012).

[12] Z. Jin, D. Gehrig, C. Dyer-Smith et al., "Ultrafast terahertz photoconductivity of photovoltaic polymer-fullerene blends: a comparative study correlated with photovoltaic device performance,” J. Phys. Chem. Lett., 5, 36623668 (2014).

[13] C. S. Ponseca, H. Nemec, N. Vukmirovic et al., "Electron and Hole Contributions to the Terahertz Photoconductivity of a Conjugated Polymer:Fullerene Blend Identified," J. Phys. Chem. Lett., 3, 2442-2446 (2012).

[14] E. Hendry, M. Koeberg, J. M. Schins et al., "Interchain effects in the ultrafast photophysics of a semiconducting polymer: THz time-domain spectroscopy of thin films and isolated chains in solution," Phys. Rev. B: Condens. Matter Mater. Phys., 71, 125201 (2005).

[15] E. Hendry, J. M. Schins, L. P. Candeias et al., "Efficiency of exciton and charge carrier photogeneration in a semiconducting polymer," Phys. Rev. Lett., 92, 196601 (2004).

[16] C. Piliego, T. W. Holcombe, J. D. Douglas et al., "Synthetic control of structural order in N-Alkylthieno[3,4c]pyrrole-4,6-dione-based polymers for efficient solar cells," J. Am. Chem. Soc., 132, 7595-7597 (2010).

[17] Y.-S. Lee, "Principles of Therahertz Scince and Technology," Springer, (2009).

[18] A. A. Paraecattil, S. Beaupre, M. Leclerc et al., "Intensity Dependent Femtosecond Dynamics in a PBDTTPDBased Solar Cell Material," J. Phys. Chem. Lett., 3, 2952-2958 (2012).

[19] M. C. Beard, G. M. Turner, and C. A. Schmuttenmaer, "Transient photoconductivity in GaAs as measured by time-resolved terahertz spectroscopy,” Phys. Rev. B: Condens. Matter Mater. Phys., 62, 15764-15777 (2000).

[20] T.-I. Jeon, D. Grischkowsky, A. K. Mukherjee et al., "Electrical characterization of conducting polypyrrole by THz time-domain spectroscopy," Appl. Phys. Lett., 77, 2452-2454 (2000).

[21] C. S. Ponseca, H. Nemec, N. Vukmirovic et al., "Electron and Hole Contributions to the Terahertz Photoconductivity of a Conjugated Polymer:Fullerene Blend Identified," J. Phys. Chem. Lett., 3, 2442-2446 (2012). 
[22] C. S. Ponseca, A. Yartsev, E. Wang et al., "Ultrafast Terahertz Photoconductivity of Bulk Heterojunction Materials Reveals High Carrier Mobility up to Nanosecond Time Scale," J. Am. Chem. Soc., 134, 11836-11839 (2012).

[23] P. D. Cunningham, and L. M. Hayden, "Carrier Dynamics Resulting from Above and Below Gap Excitation of P3HT and P3HT/PCBM Investigated by Optical-Pump Terahertz-Probe Spectroscopy,” J. Phys. Chem. C, 112, 7928-7935 (2008).

[24] J. Lloyd-Hughes, and T.-I. Jeon, "A review of the terahertz conductivity of bulk and nano-materials," J. Infrared, Millimeter, Terahertz Waves, 33, 871-925 (2012).

[25] F. C. Grozema, P. T. van Duijnen, Y. A. Berlin et al., "Intramolecular charge transport along isolated chains of conjugated polymers: effect of torsional disorder and polymerization defects," J. Phys. Chem. B, 106, 77917795 (2002).

[26] P. Parkinson, J. Lloyd-Hughes, M. B. Johnston et al., "Efficient generation of charges via below-gap photoexcitation of polymer-fullerene blend films investigated by terahertz spectroscopy," Phys. Rev. B: Condens. Matter Mater. Phys., 78, 115321 (2008).

[27] K. P. H. Lui, and F. A. Hegmann, "Ultrafast carrier relaxation in radiation-damaged silicon on sapphire studied by optical-pump-terahertz-probe experiments," Appl. Phys. Lett., 78, 3478-3480 (2001).

[28] T. M. Burke, and M. D. McGehee, "How high local charge carrier mobility and an energy cascade in a threephase bulk heterojunction enable >90\% quantum efficiency," Adv. Mater., 26, 1923-1928 (2014).

[29] O. G. Reid, J. A. Nekuda Malik, G. Latini et al., "The influence of solid-state microstructure on the origin and yield of long-lived photogenerated charge in neat semiconducting polymers," J. Polym. Sci., Part B: Polym. Phys., 50, 27-37 (2012).

[30] X. Ai, M. C. Beard, K. P. Knutsen et al., "Photoinduced charge carrier generation in a poly(3-hexylthiophene) and methanofullerene bulk heterojunction investigated by time-resolved terahertz spectroscopy," J. Phys. Chem. B, 110, 25462-25471 (2006). 